Published in final edited form as:

ACS Appl Mater Interfaces. 2019 May 08; 11(18): 16380-16390. doi:10.1021/acsami.9b04051.

\title{
pH-Responsive Nanoparticles Targeted to Lungs for Improved Therapy of Acute Lung Inflammation/Injury
}

\author{
Can Yang Zhang ${ }^{\dagger}$, Wenjing Lin ${ }^{\ddagger}$, Jin Gao ${ }^{\dagger}$, Xutong Shi $^{\dagger}$, Maryam Davaritouchaee ${ }^{\#}$, Amy E. \\ Nielsen\#, Rock J. Mancini ${ }^{\#}$, and Zhenjia Wang ${ }^{\dagger,}{ }^{*}$ \\ † Department of Pharmaceutical Sciences, College of Pharmacy and Pharmaceutical Sciences, \\ Washington State University, Spokane, WA 99210, United States \\ ‡ School of Chemical Engineering and Light Industry, Guangdong University of Technology, \\ Guangzhou 510006, China \\ \# Department of Chemistry, Washington State University, Pullman, WA 99164, United States
}

\begin{abstract}
Dysregulated vascular inflammation is the underlying of acute lung inflammation/injury (ALI). Bacterial infections and trauma cause ALI that may rapidly lead to acute respiratory distress syndrome (ARDS). There are no pharmacological therapies available to patients with ALI/ARDS, partially as drugs cannot specifically target the lungs. Herein, we developed a stimuli-responsive nanoparticle to target inflammatory lungs for ALI therapies. The nanoparticle is comprised of a sharp acid-sensitive segment poly $(\beta$-amino esters) (PAE) as a core for drug loading and controlled release, and a polyethylene glycol (PEG)-biotin on the particle surface available for bioconjugation enabling lung targeting and extended circulation. The studies on dissipative particle dynamics (DPD) simulation and characteristics of nanoparticles (NPs) suggest that antiICAM-1 antibodies can be coated to the particle surface and this coating is required to enhance lung targeting of NPs. A model drug of anti-inflammatory agent TPCA-1 is encapsulated in NPs with high drug loading content at $24 \%(\mathrm{w} / \mathrm{w})$. In the mouse ALI model, our TPCA-1-loaded NPs coated with anti-ICAM-1 can target inflamed lungs after intravenous injection, followed by drug release triggered by acid environment, thus mitigating lung inflammation and injury. Our studies reveal the rational design of nanotherapeutics for improved therapy of ALI and it may be applied to treating a wide range of vascular inflammation.
\end{abstract}

\section{Keywords}

acute lung inflammation; pH-responsive; nanoparticles; targeted drug delivery; controlled release

*Corresponding author: Zhenjia Wang: zhenjia.wang@wsu.edu.

Conflict of Interest

The authors declare no conflict of interest. 


\section{Introduction}

Inflammation is the immune response to infections and tissue injury to protect the body from damage. However, uncontrolled inflammation is the cause of many acute and chronic diseases. ${ }^{1,2}$ For example, bacterial infections and trauma can cause acute lung inflammation/injury (ALI), that may rapidly precipitate acute respiratory distress syndrome (ARDS). ${ }^{3,4}$ The cases of ALI/ARDS are more than 200,000 annually in the USA, and it is also growing worldwide. ${ }^{4}$ Although advanced technologies have been developed in lungprotection ventilation and fluid management, the mortality of ALI/ARDS is still high at 30$50 \%{ }^{3,4}$ However, there are no effective therapeutics available to ALI/ARDS patients due to insufficient drug delivery to the lungs. ${ }^{5-8}$

Significant research efforts have harnessed on developing delivery strategies to improve treatments of lung diseases using nanotechnology in recent years. ${ }^{9-14}$ For example, bacterial membrane-derived nanovesicles have been developed for vaccination to prevent bacteriuminduced lung infection because the nanovesicles contain antigens of invaded bacteria. ${ }^{15,16} \mathrm{In}$ addition, various nanoparticle-based drug delivery systems, such as polymeric micelles, lipid and nanogel have been used to enhance drug delivery to the lungs. ${ }^{17-20}$ For instance, Kaczmarek et al. reported a pH-sensitive polymer-lipid hybrid nanoparticle that was capable of delivering mRNA to the mouse lungs after intravenous administration, ${ }^{21}$ but the mechanism by which the NPs were preferential deposition in the lungs remains unknown. Anselmo et al. prepared a red blood cell (RBC)-based drug delivery system to increase accumulation of nanoparticles in the lungs because of the prolonged circulation of NPs. ${ }^{22}$

Dysregulated inflammation and increased lung permeability are the features of ALI/ARDS. ${ }^{23}$ In the early stage of ALI, vascular inflammation initiates transmigration of neutrophils (white blood cells in circulation) into the lungs involved with interactions of neutrophils with activated endothelia. ${ }^{24-26}$ The binding of integrin $\beta_{2}$ on neutrophils to intercellular adhesion molecule-1 (ICAM-1) on endothelial cells promotes neutrophil transmigration. ${ }^{27}$ Furthermore, inflammatory microenvironment (IME) has the unique features including low $\mathrm{pH}$, excessive enzymes and increased body temperature. ${ }^{28,} 29$ Inspired by these unique features in IME, here we designed and prepared anti-ICAM-1-coated nanoparticles (NPs) that are able to target inflammatory lung endothelia and deliver anti-inflammatory agents in the manner of controlled release triggered by low $\mathrm{pH}$ existing in IME, thus possibly reversing the progression of lung inflammation/injury.

Scheme 1 shows the hypothesis of our study. The amphiphilic copolymers comprised of $\mathrm{pH}-$ sensitive hydrophobic segment PAE and hydrophilic segment PEG-biotin can self-assemble into polymeric micelles. To target inflammatory lung endothelia, we design the copolymers of both terminals capped with biotin for coating of anti-ICAM-1 molecules. (2[(Aminocarbonyl)amino]-5-(4-fluorophenyl)-3-thiophenecarboxamide (TPCA-1) is used as a model drug because TPCA- 1 is an anti-inflammatory agent. ${ }^{30}$ TPCA-1 is a potent and selective inhibitor of I $k$ B kinase-2 (IKK-2) with IC50 $=17.9 \mathrm{nM}$ for IKK-2 compared to $400 \mathrm{nM}$ for IKK-1. ${ }^{31}$ TPCA-1 can inhibit the production of TNF-alpha, IL-6, and IL-8 from human monocytes after they were treated with lipopolysaccharide (LPS). ${ }^{30}$ TPCA-1 is insoluble in water and does not have the tissue targeting feature. To improve the delivery 
efficacy of TPCA-1 to the inflammatory lung, TPCA-1 is encapsulated in polymeric micelles (called TPCA-1-PMs). We hypothesized that biotin may be exposed on the surface of TPCA-1-PMs for bioconjugation. Anti-ICAM-1 may be easily coated to NPs via interaction between biotin and avidin (TPCA-1-NPs-anti-ICAM-1 is called endothelial targeted nanotherapeutics). ${ }^{32-34}$ When they are intravenously (i.v.) administered to a mouse with acute lung inflammation, NPs may specifically target to the inflamed lung endothelia and rapidly release the drug, thus improving the treatment of ALI.

\section{Experimental Section}

\subsection{Materials}

$O$-(2-Aminoethyl)- $O^{\prime}$-[2-(biotinylamino)ethyl]octaethylene glycol (Biotin-PEG-NH $\mathrm{NH}_{2}, \geq$ $95 \%, M_{\mathrm{w}}=3400$ ), 1,6-hexanediol diacrylate (HDD, $99 \%$ ), 3-amino-1-propanol (AP, $99 \%$ ), TPCA-1 ( $\geq 95 \%$, [5-(p-Fluorophenyl)-2-ureido]thiophene-3-carboxamide) and lipopolysaccharide (LPS, Escherichia coli 0111:B4) were purchased from Sigma-Aldrich (St. Louis, MO). Cyanine 5 NHS ester (Cy5-NHS) was obtained from Lumiprobe Co., USA. Fluorescein isothiocyanate (FITC) was purchased from Alfa Aesar. NeutrAvidin ${ }^{\mathrm{TM}}$ biotin binding protein was purchased from Thermo Fisher Scientific. All chemical reagents were used as received. Biotinylated rat IgG2b and biotinylated anti- ICAM-1 antibodies (Abs) were purchased from Biolegend (San Diego, CA). Pierce ${ }^{\text {TM }}$ BCA protein assay kit was purchased from Thermo Fisher Scientific. All cell lines, biological reagents and other cell culture medium were used as received following our previous protocols. ${ }^{13}$

\subsection{Synthesis of Copolymer}

The copolymer was synthesized via Michael-type polymerization. Acrylate-terminated PAE was synthesized as described in the previous report with a few modifications. ${ }^{35-37}$ Briefly, HDD $(1.1 \mathrm{mmol})$ and AP $(1.0 \mathrm{mmol})$ were dissolved in anhydrous DMSO in a $50 \mathrm{~mL}$ Schlenk flask and stirred constantly. After evacuation-flush with nitrogen three times, the flask was immersed into silicone oil and heated to $90{ }^{\circ} \mathrm{C}$. The reaction was carried out for 4 $\mathrm{h}$ with gentle stirring. The mixture was dissolved in DCM after it was cooled down, and the resulted solution was precipitated with $n$-hexane three times. The designed acrylate-ended PAE was received after drying under vacuum for $24 \mathrm{~h}$. Next, amine-ended Biotin-PEG-NH was conjugated on the terminals of PAE. In brief, Biotin-PEG-NH 2 and PAE (2: 1 molar ratio) were dissolved in anhydrous DMSO. The reaction was carried out for 3 days at $65{ }^{\circ} \mathrm{C}$ under nitrogen. After purification and precipitation with $n$-hexane three times, the designed Biotin-PEG-PAE-PEG-Biotin polymer was obtained.

For FITC-labeled copolymer, ${ }^{38}$ the copolymer $(400 \mathrm{mg}$ ) was dissolved into $20 \mathrm{~mL}$ of anhydrous DMSO, and then $4 \mathrm{mg}$ FITC was added into the solution. The resulting solution was reacted for $4 \mathrm{~h}$ at $90{ }^{\circ} \mathrm{C}$ in the dark. The solution was dialyzed (MWCO: 3500-4000 Da) against distilled water for 3 days. The FITC-labeled copolymer was obtained after lyophilization. For Cy5-labeled TPCA-1, Cy5-NHS and TPCA-1 (1:1, molar ratio) were dissolved, and the reaction was carried out for 5 days. After purification and lyophilization, Cy5-labeled TPCA-1 was received. 


\subsection{Preparation of NPs}

TPCA-1-loaded polymeric micelles (TPCA-1-PMs) were prepared using dialysis method. ${ }^{37}$ Briefly, the copolymer $(40 \mathrm{mg})$ was dissolved in DMF $(40 \mathrm{~mL})$, followed by addition of different amount of TPCA-1 (5 mg, $10 \mathrm{mg}, 20 \mathrm{mg}$ ). As reported in our previous work, ${ }^{37}$ the solution was filtered and lyophilized, and the powder was obtained for storage.

To coat antibodies to drug-loaded NPs, TPCA-1-PMs were suspended in deionized water, followed by the stepwise addition of avidin under stirring. 5 min later, the suspension was centrifuged at $15,000 \mathrm{~g}$ for $5 \mathrm{~min}$ at $4{ }^{\circ} \mathrm{C}$ to remove free avidin, resulting in avidin-decorated PMs. Biotinylated rat IgG2b or biotinylated anti-ICAM-1 antibody $\left(2 \mathrm{~mL}, 0.1 \mathrm{mg} \mathrm{mL}^{-1}\right.$, PBS, pH 7.4) was added to avidin-decorated PMs (2 mL, $0.2 \mathrm{mg} \mathrm{mL}^{-1}$, PBS, pH 7.4) under stirring (1000 rpm) at room temperature. 20 min later, the suspension was centrifuged at $15,000 \mathrm{~g}$ for $5 \mathrm{~min}$ at $4{ }^{\circ} \mathrm{C}$ to remove free antibody, and then the pellet was re-suspended in $\mathrm{PBS}$ at $\mathrm{pH}$ 7.4. The supernatant was collected for bicinchoninic acid assay (BCA) assay. The antibody-coated NPs were characterized by sodium dodecyl sulfate polyacrylamide gel electrophoresis (SDS-PAGE). The FITC and Cy5-labeled NPs were prepared similarly.

\subsection{Characterization}

${ }^{1} \mathrm{H}$ NMR spectra of samples were recorded by a Bruker AVANCE III 500 (Switzerland) spectrometer operating at $250 \mathrm{MHz}$ at $25^{\circ} \mathrm{C}$ The solvent was deuterated chloroform $\left(\mathrm{CDCl}_{3}-d\right)$. The number-average molecular weight $\left(M_{\mathrm{n}}\right)$ and polydispersity index (PDI, $M_{\mathrm{w}} / M_{\mathrm{n}}$ ) were determined by gel permeation chromatography (GPC) with a Waters 1525 chromatograph and a Waters 2414 refractive index (RI) detector. THF was used as eluent, and the flow rate was $1.0 \mathrm{~mL} / \mathrm{min}$.

High performance liquid chromatography (HPLC) was performed using a Waters 2690 Separations Module system (Milford, MA), including a P-900 gradient pump system, a Waters 486 Tunable absorbance detector and a Ultra C18 $5 \mu \mathrm{m}$ column $(250 \times 4.6 \mathrm{~mm})$. The measurement was carried out using acetonitrile/water $(70: 30, \mathrm{v} / \mathrm{v})$ as the mobile phase with a flow rate of $1.0 \mathrm{~mL} / \mathrm{min}$ and detected at a wavelength of $310 \mathrm{~nm}$.

The critical micelle concentration (CMC) of copolymer was measured by the fluorescence technique using pyrene as a fluorescence probe. The process was described in detail in our previous work. ${ }^{37}$

The particle size and distribution (polydispersity index, PDI) were determined by dynamic light scattering (DLS) with a Malvern Zetasizer Nano90 (Westborough, MA). The sample was prepared in a $1.0 \mathrm{~mL}$ quartz cuvette, and the measurements were conducted using a diode laser of $670 \mathrm{~nm}$ at room temperature with the scattering angle $90^{\circ}$. In addition, the particle size of sample which was dispersed in PBS containing $20 \%$ FBS at the normal physiological $\mathrm{pH}$ after incubation at $37^{\circ} \mathrm{C}$ was also measured by DLS.

Morphologies of various NPs were investigated by transmission electron microscopy (TEM) (FEI Technai G2 20 Twin TEM (Hillsboro, Oregon)). 
The LC (loading content) and EE (encapsulation efficiency) of NPs were measured by HPLC as aforementioned. Briefly, TPCA-1-loaded NPs (1 mg) were completely dissolved in $1 \mathrm{~mL}$ methanol. HPLC analysis was performed, and the amount of drug was calculated according to the standard curve. And then, $\mathrm{LC}$ and EE were calculated according to the following equations (1) and (2), respectively:

$$
\begin{gathered}
L C(\%)=\frac{\text { Weightofloadeddrug }}{\text { Weight ofdrugloaded }-N P S} \times 100 \% \\
E E(\%)=\frac{\text { Weightofloadeddrug }}{\text { Weightofdruginfeed }} \times 100 \%
\end{gathered}
$$

\subsection{Dissipative Particle Dynamics (DPD) Simulation}

The self-assembly of copolymer and the distribution of drug were studied by DPD simulation based on a coarse-grained model. ${ }^{39}$ DPD is a stochastic coarse-grained simulation method suitable for complex multiphase systems, in which molecules are divided into a set of soft interacting beads. Each bead represents a group of atoms or a volume of fluid. Firstly, polymer molecule was divided into four types of beads: A (peach, methylene groups from HDD), B (green, repeat units from PEG), C (gross green, biotin), D (pink, AP). TPCA-1 molecule was divided into two types of beads (blue). And three water molecules were considered a bead of Water (black). The interaction parameters $\left(a_{i j}\right)$ were calculated based on our previous method and DPD simulation was conducted in the Mesocite module of Materials Studio 5.0 software (Accelrys Inc., USA), ${ }^{39,40}$ as shown in Table S1. And the calculation details were shown in Supporting Information. A cubic simulation box of $30 \times 30 \times 30 r_{\mathrm{c}}{ }^{3}$ with periodic boundary condition was applied in all three directions. The simulation steps are 100,000 with an integration time step was $0.05 \mathrm{~ns}$.

\subsection{Drug Release Profile In Vitro}

The in vitro release profile of TPCA- 1 was studied using dialysis method at $37^{\circ} \mathrm{C}$. Briefly, 5 mg of TPCA-1 loaded NPs was dispersed in $5 \mathrm{~mL}\left(V_{\mathrm{e}}\right) \mathrm{PBS}$ at $\mathrm{pH} 7.4$ or 6.5. The solution was placed in a dialysis bag (MWCO: $3500-4000 \mathrm{Da}$ ) which was immersed in $45 \mathrm{~mL}$ PBS at $\mathrm{pH} 7.4$ or 6.5 in a beaker. The beaker was then placed in a $37^{\circ} \mathrm{C}$ water bath with stirring at $110 \mathrm{rpm} .1 \mathrm{~mL}$ solution was drawn at pre-determined timepoint for HPLC analysis and 1 $\mathrm{mL}$ fresh buffer was added. The cumulative drug release percentage $\left(E_{\mathrm{r}}\right)$ was calculated based on the equation (3):

$$
E_{\mathrm{r}}(\%)=\frac{V_{e} \sum_{1}^{n-1} C_{\mathrm{i}}+V_{0} C_{\mathrm{n}}}{m_{\mathrm{drug}}} \times 100
$$

where, $m_{\text {drug }}$ is the weight of loaded TPCA- $1, V_{0}$ is the volume of solution in a beaker ( $V_{0}=$ $50 \mathrm{~mL}$ ), and $C_{\mathrm{i}}$ is the concentration of TPCA- 1 in the $i_{\mathrm{th}}$ sample. The experiment was repeated three times. 


\subsection{Cell Culture}

Human vascular endothelial cells (HUVECs) were cultured and incubated according to the report in our lab. ${ }^{13}$ The cells were activated using $50 \mathrm{ng} / \mathrm{mL}$ TNF- $a$ (Biolegend, San Diego, CA) with incubation for 4 hours. The cells only within 7 passages were used for experiments.

\subsection{Cell Cytotoxicity}

Cell cytotoxicity of copolymer was measured by a CCK- 8 assay method. HUVECs were firstly passaged and plated in 96-well plates at an initial density of 5,000-10,000 cells/well and cultured for $24 \mathrm{~h}$ in a humidified atmosphere containing $5 \% \mathrm{CO}_{2}$. Polymeric micelle solution was prepared with a completed medium at different concentrations. After removing the culture supernatant, $2001 / 4 \mathrm{~L}$ of fresh medium or pre-prepared sample solution was added in every well. $48 \mathrm{~h}$ after incubation, $10 \mu \mathrm{L}$ of the cell proliferation reagent (Promega, Madison, WI) was added in every experimental well. After incubation for $2 \mathrm{~h}$, the absorption at $490 \mathrm{~nm}$ was measured by a microplate reader. All experiments were performed in hexaplicate.

\subsection{Binding of NPs to HUVEVs In Vitro}

HUVECs were seeded in a $3.5 \mathrm{~cm}$ dish and incubated for $24 \mathrm{~h}$, and then treated with or without TNF- $a$. The cells were then washed with PBS for three times, and incubated in PBS with or without anti-ICAM-1 antibody for $30 \mathrm{~min}$. After fluorescently-labeled Abs-coated drug-loaded NPs were added, the sample was incubated for $30 \mathrm{~min}$ at room temperature. After washing with PBS for three times, the cells were analyzed by Accuri C6 flow cytometer (BD Biosciences, San Jose, CA). For imaging, the resulted samples were treated with paraformaldehyde (PFA, $2 \mathrm{~mL}, 4 \%$ ) for $30 \mathrm{~min}$ on ice, and the slide smears were prepared with 7620 Cytopro Cytocentrifuge (ELITech, Princeton, NJ). One drop of Prolong Gold anti-fade reagent with DAPI (Invitrogen, Eugene, OR) was added on the cells, and a cover slip was applied on the slide. The cells were imaged by a Nikon $\mathrm{A} \mathrm{R}^{+}$laser scanning confocal microscope.

\subsection{Mouse Experiments}

Adult CD-1 mice (18-25 g) were purchased from Harlan Labs (Madison, WI). All animal care and experimental protocols used in the studies have been approved by the Washington State University Institutional Animal Care and Use Committee. Mouse maintenance and care followed the description in our previous work. ${ }^{13}$

\subsection{Acute Lung Inflammation Mouse Model}

The acute lung inflammation mouse model was established as reported by our lab. ${ }^{5}$ Mice were anesthetized firstly, and then they were fixed in a supine position for administration of LPS to the lung. $15 \mathrm{mg} / \mathrm{kg}$ of LPS in 75 1/4L of Hank's Balanced Salt Solution (HBSS) was nebulized into pulmonary alveoli with an FMJ-250 High Pressure Syringe (Penn-Century, Wyndmoor, PA). $4 \mathrm{~h}$ later, mice were grouped randomly and $i . v$. administered with PBS, free TPCA-1 (2 mg/kg), two types of Abs-coated TPCA-1-NPs (equal to $2 \mathrm{mg} / \mathrm{kg}$ of TPCA-1), respectively. $20 \mathrm{~h}$ later, mice were anesthetized, and the trachea was cannulated. $1 \mathrm{~mL}$ HBSS 
was i.t. infused to the lung and withdrawn back to collect lung bronchoalveolar lavage fluid (BALF). This procedure was repeated three times. After centrifugation at $350 \mathrm{~g}$ for $10 \mathrm{~min}$, the supernatant was collected for ELISA analysis. The pelleted cells were collected and the cell numbers were counted on a hemocytometer.

\subsection{In Vivo Biodistribution of NPs}

Acute inflammation lung mouse model was generated as mentioned above. $4 \mathrm{~h}$ later, the mice were grouped randomly and i.v. injected with fluorescent TPCA-1-NPs-Abs or free TPCA-1. At the pre-determined time intervals, lungs were collected and imaged using in vivo imaging systems (IVIS) (Caliper Life Sciences, Waltham, MA) at 640/680 nm (for Cy5) and 480/520 nm (for FITC).

\subsection{Protein Content Determination}

The coating rate of antibody on NPs and protein concentrations in supernatant of BALF were determined by the BCA method using a commercial kit according to the protocol.

\subsection{ELISA Assay}

The contents of inflammatory factor (IL-6 and TNF- $a$ ) in the supernatant of BALF were measured with ELISA MAX Deluxe Sets according to the protocol.

\subsection{Hematoxylin and Eosin (H\&E) Stain}

After mice were treated with PBS, free drug, two types of Abs-coated NPs loaded with drug in ALI mice, the mice were euthanized by carbon dioxide. Lungs were removed and fixed with $10 \%$ formalin, embedded in paraffin, sectioned at $5 \mu \mathrm{m}$ and stained with hematoxylin and eosin for pathology studies using RTPH 360 Rapid Tissue Processor Operator Manual and SS-2030 Linear Slide Stainer. The samples were imaged by fluorescence confocal microscopy (ZEISS, Observer. Z1, USA).

\subsection{Statistical Analysis}

Experimental data were presented as mean \pm standard deviation (s.d.). Statistical analysis was conducted using one-way ANOVA or Student's $t$-test of Origin 8.5, $p$ values $<0.05$ were considered significant.

\section{Results and Discussion}

\subsection{Synthesis and Characterization of Copolymer Biotin-PEG-b-PAE-b-PEG-Biotin}

The $\mathrm{pH}$-sensitive and biotin-coated amphiphilic block copolymer Biotin-PEG- $b$-PAE- $b$ PEG-Biotin was synthesized as shown in Figure S1 (Supporting Information). The acrylateended poly ( $\beta$-amino esters) (PAE) was firstly generated via Michael-type addition polymerization, ${ }^{37,41}$ and then PEG-biotin was conjugated on the terminals of PAE, resulting in designed block copolymer. The chemical structures of PAE and polymer were confirmed by ${ }^{1} \mathrm{H}-\mathrm{NMR}$ (Figure 1A and B). As shown in Figure 1A, the signals at 5.8-6.5 ppm (a, b and c) were characteristic peaks of three protons $\left(\mathrm{H}_{2} \mathrm{C}=\mathrm{CH}\right.$-) on the terminals of PAE. The signals at $4.1 \mathrm{ppm}(\mathrm{d}), 1.6 \mathrm{ppm}(\mathrm{e})$ and $1.2 \mathrm{ppm}$ (f) were ascribed to the $-\mathrm{CH}_{2}-\mathrm{O},-\mathrm{CH}_{2}-\mathrm{CH}_{2-}$ 
$\mathrm{O}$ and $-\mathrm{CH}_{2}$ - in the main polymer chain, respectively. The signals at $2.5 \mathrm{ppm}(\mathrm{g})$ and 2.7 ppm (h) were, respectively, due to the vibration of $-\mathrm{CH}_{2}-\mathrm{C}=\mathrm{O}$ and $-\mathrm{CH}_{2}-\mathrm{N}$. The three characteristic peaks of $2.6 \mathrm{ppm}$ (i), $1.5 \mathrm{ppm}(\mathrm{j})$ and $3.7 \mathrm{ppm}(\mathrm{k})$ were assigned to $\mathrm{N}-\mathrm{CH}_{2^{-}}$, $\mathrm{CH}_{2}$ - and $-\mathrm{CH}_{2}-\mathrm{OH}$ on the tail. As shown in Figure 1B, the characteristic three peaks of acrylate-ended PAE ( $a, b$ and $c$ ) were disappeared, indicating the successful conjugation of PEG-biotin on terminals of PAE. Furthermore, the characteristic peaks of ring in biotin ( $a, b$, $\mathrm{c}$ and d) and the characteristic peak of PEG (3.6 ppm, m) was observed. All results demonstrated that the copolymer Biotin-PEG- $b$-PAE- $b$-PEG-Biotin was successfully synthesized. The molecular weight and PDI of PAE and copolymer were confirmed as 4320 $\mathrm{g} / \mathrm{mol}$ (PDI 2.12) and $9045 \mathrm{~g} / \mathrm{mol}$ (PDI 2.35), respectively by gel permeation chromatography (GPC, Supporting Information, Figure S2). A critical micelle concentration (CMC) of copolymer was measured by pyrene fluorescence spectroscopy and found that it was $7.91 / 4 \mathrm{~g} / \mathrm{mL}$ (Figure 1C), demonstrating that the copolymer could self-assemble into a micelle at low copolymer concentrations.

To determine the $\mathrm{pH}$ response of our copolymer, $\mathrm{p} K_{\mathrm{b}}$ value was measured by a potentiometric titration (Figure 1D). With addition of $\mathrm{NaOH}$ solution, the $\mathrm{pH}$ value was increased sharply and then reached a plateau (6.5-6.9), due to the protonation/deprotonation of tertiary amine groups in PAE segments. The $\mathrm{p} K_{\mathrm{b}}$ value of copolymer was determined to be 6.7 based on the $\mathrm{pH}$ value at a half of neutralization of amine protonation, ${ }^{42,}{ }^{43}$ showing that the copolymer was responsive to acid. Furthermore, the sizes of NPs at different $\mathrm{pH}$ were measured by DLS (Supporting Information, Figure S3). We observed that the size of nanoparticle increased when the $\mathrm{pH}$ decreased from 7.4 to 3.0, because tertiary amine residues in PAE segment of the micelle were protonated and the nanoparticles were swollen. This result indicated that NPs were responsive to $\mathrm{pH}$ and this effect may promote drug release.

\subsection{Preparation and Characterization of NPs}

Next, we addressed whether biotin is exposed on the surface of NPs because this is required for biofunctionalization of NPs. Firstly, the dissipative particle dynamics (DPD) simulation was used to evaluate whether the biotin segments distribute on the surface of NPs. The copolymer, the model drug (TPCA-1) and water were coarse-grained as shown in Figure 2A. In DPD simulation, copolymer chains and TPCA-1 molecules were randomly dispersed in water, and we monitored their movements. We observed that copolymer and TPCA-1 molecules gradually co-self-assembled into drug-loaded polymeric micelles (TPCA-1-PMs) after 100,000 steps (Figure 2B). The results were consistent with the CMC measurement, indicating the copolymers were able to self-assemble into micelle together with model drug TPCA-1. Figure 2C showed a full view and a cross section of TPCA-1-PMs where PAE (red) moieties and PEG (light green) moieties formed an inner micellar core and a shell on the surface, respectively. The drug TPCA-1 (blue) molecules were entrapped into the core due to hydrophobic interactions. The result showed that the TPCA-1-loaded polymeric micelle possessed a typical core-shell structure and the TPCA-1 was entrapped in the micellar core. Furthermore, the biotin segments (grass green) were exposed on the surface of nanoparticle which was required for conjugation of Abs to the NPs. To confirm the conjugation of Abs to the NPs, we characterized the morphology of TPCA-1-PMs and 
TPCA-1-NPs-Abs (Figure 2D and E) using transmission electron microscopy (TEM). When we closely examined the NPs coated with anti-ICAM-1, we found a thin corona around the nanoparticle (Figure 2E) compared with that without antibody decoration (Figure 2D), suggesting that coating of anti-ICAM-1 to the surface of NPs was achieved.

Next, sodium dodecyl sulfate polyacrylamide gel electrophoresis (SDS-PAGE, Figure 2A) showed that anti-ICAM-1 antibodies (Abs) were successfully linked on the surface of NPs. The conjugation efficiencies (CE) of antibodies (Abs) were measured by bicinchoninic acid assay (BCA, Figure 2B). The CE for anti-ICAM-1 and negative control IgG2b was $40 \%$ and $37 \%$, respectively, , suggesting that the bio-conjugation was independent on antibodies. Collectively, computer simulation and experimental data indicated that we can link Abs to the particle surface via biotin-avidin interaction. Moreover, the TPCA-1 loading content (LC) and encapsulation efficacy (EE) were determined at different ratios of copolymer to drug (Supporting Information, Table S2). We found that our NPs can efficiently load TPCA-1 at 10-24\% (w/w). In addition, the ratio for copolymer/drug at 40/10 offered the optimal LC and EE of TPCA-1 in NPs, so we chose this formulation in the rest studies.

In addition, we measured the particle sizes using dynamic light scattering (DLS), showing that TPCA-1-NPs-Abs slightly increased their size to $100 \mathrm{~nm}$ (polydispersity index 0.21 ) compared to $82.2 \mathrm{~nm}$ (polydispersity index 0.18 ) of NPs without antibody coating (Supporting Information, Figure S4). This result is consistent with TEM images (Figure 2F and $\mathrm{G}$ ). Combining the computer simulation with experiments demonstrates that biotin is exposed on the surface of PMs after amphiphilic copolymer is self-assembled. The serum stability of drug-loaded PMs, two types of Abs-coated NPs with drug loading was evaluated by measurement of hydrodynamic diameter after their incubation in 4 days (Supporting Information, Figure S5). The results showed three of nanoparticles exhibited high stability, thus they may be a good delivery carrier.

\subsection{NPs Target Inflamed Endothelium}

To address whether TPCA-1-NPs-Abs can target inflammatory lung endothelium, NPs and TPCA-1 were labeled by FITC and Cy5, respectively. First, we studied how NPs interacted with human umbilical vein cells (HUVECs) (Figure 3A, Supporting Information, Figure S6). When HUVECs were not activated using TNF- $a$, no fluorescence of FITC and Cy5 were observed after their incubation with endothelial targeted nanotherapeutics which was decorated with anti-ICAM-1. When we treated HUVECs using TNF- $a$ followed by the successive incubation with anti-ICAM-1 and endothelial targeted nanotherapeutics, we did not observe the fluorescence from NPs and TPCA-1 either. In another control, we activated HUVECs and incubated them with non-endothelial targeted nanotherapeutics (TPCA-1NPs-IgG2b) which was decorated with IgG2b. We observed no binding of NPs to HUVECs. However, we observed the strong fluorescence of both NPs and TPCA-1 when the HUVECs were activated with TNF- $a$ followed by incubation with endothelial targeted nanotherapeutics. In order to quantitatively analyze the binding/uptake of NPs by HUVECs, we performed flow cytometry to measure the percentage of nanoparticle uptake (Figure 3B) and mean fluorescence intensity (MFI) (Figure 3C). After HUVECs were activated by TNF$a$ and they were incubated with endothelial targeted nanotherapeutics, we observed that the 
percentages of nanoparticle uptake were increased (Figure 3B) and the MFI was accordingly increased (Figure 3C). The results showed that anti-ICAM-1 decoration on the surface of NPs promoted the binding/uptake of nanoparticles. This is consistent with the confocal fluorescence images (Figure 3A).

Next, we address whether the inflammation increases the penetration of nanoparticles across a monolayer of HUVEVs. To do so, we established a Transwell assay (Figure 3D). Endothelial cells were grown on the porous membrane to form a monolayer and they were treated with TNF- $a$ to cause inflammation response. One hour after the addition of NPs to the upper chamber, trafficking of NPs to the lower chamber was measured. Without TNFatreatment, the transport of NPs was not dependent on ICAM-1 coating to NPs. However, after TNF- $a$ activation, we observed the more NPs in the low chamber compared to that without TNF- $a$ treatment (Figure 3E), implying that endothelial inflammation facilitated the transport of NPs. The molecular mechanism is complicated and it may be associated with increased junction of endothelial layer and possibly the cell death to promote the transport of NPs. ${ }^{23}$ In addition, endothelial targeted nanotherapeutics in the lower chamber was increased by 2 folds compared to NPs-IgG2b (Figure 3E), indicating that anti-ICAM- 1 decoration promoted the binding of nanoparticles to inflamed vascular endothelial cells for enhanced intercellular trafficking.

\subsection{Anti-ICAM-1-coating Promotes Lung Delivery of NPs}

Next, we ask whether endothelial targeted nanotherapeutics can specifically target to inflammatory mouse lungs in vivo. The mouse was challenged by lipopolysaccharides (LPS) which is a toxin derived from the outer membrane of Gram-negative bacteria. ${ }^{6}$ Figure $4 \mathrm{~A}$ is the protocol of animal experiment. Firstly, mice were intratracheally (i.t.) challenged with LPS $(10 \mathrm{mg} / \mathrm{kg})$ to induce acute lung inflammation. Fluorescent TPCA-1-NPs-Abs were then intravenously (i.v.) administered to visualize the trafficking of NPs and the drug in the lungs. $6 \mathrm{~h}, 16 \mathrm{~h}$ and $24 \mathrm{~h}$ after $i . v$. administration of TPCA-1-NPs-Abs or free drug, the lungs were collected and imaged by in vivo imaging systems (IVIS). Figure 4B and C showed the accumulation of NPs and the drug in lung, respectively. We observed that both NPs and the drug (TPCA-1) showed the strong fluorescence in endothelial targeted nanotherapeutics- treated group compared to those in non-endothelial targeted nanotherapeutics- and free drug-treated groups, suggesting that anti-ICAM-1 decoration on the surface of NPs is required for improved drug delivery in inflammatory lungs. We still observed the fluorescence of both NPs and drug in endothelial targeted nanotherapeutics treated group for a long period $(24 \mathrm{~h})$ although there was a trend of decreased fluorescence. The decreased fluorescence may be associated with fluorescence quenching and degradation of labeled dyes. The quantitative analysis of IVIS demonstrated that the accumulation of anti-ICAM-1-coated NPs in the lungs was increased by 2 folds compared to the negative control $2 \mathrm{~h}$ after administration of NPs (Figure 4D), and we could observe the NPs in the lungs over $20 \mathrm{~h}$. We also monitored the trafficking of TPCA-1, as shown in Figure 4E. Free TPCA-1 was hardly observed in the inflammatory lung. When the drug-loaded NPs were $i . v$. administrated, TPCA-1 in lung was increased. Particularly, we observed that the accumulation of TPCA- 1 in lung for mice treated with endothelial targeted nanotherapeutics was 5-fold and 3-fold higher than that for the negative control at $6 \mathrm{~h}$ and $24 \mathrm{~h}$, respectively.

ACS Appl Mater Interfaces. Author manuscript; available in PMC 2020 May 08. 
The results indicate that anti-ICAM-1decoration to nanoparticles significantly enhances the lung deposition of nanoparticles, thus resulting in the increased TPCA-1 in the lungs.

\subsection{Improved ALI Therapy}

To address the usefulness of our newly-developed nanoparticle-based drug formulations, we delivered TPCA-1 to mice in the acute lung inflammation model. Firstly, as shown in Figure $5 \mathrm{~A}$, the drug release profile of endothelial targeted nanotherapeutics in vitro at various $\mathrm{pH}$ values was measured to evaluate the $\mathrm{pH}$-triggered TPCA-1 release property. At $\mathrm{pH} 7.4$, the accumulative release of TPCA-1 was less than $20 \%$ at $24 \mathrm{~h}$, however it was increased to approximately $90 \%$ at $\mathrm{pH} 6.5$ at $15 \mathrm{~h}$. The results indicated that TPCA- 1 could be quickly released from NPs in acidic tissues, suggesting that TPCA-1 release from nanocarriers was dependent on $\mathrm{pH}$ value. Figure 5B shows the experimental protocol. $4 \mathrm{~h}$ after LPS (15 $\mathrm{mg} / \mathrm{kg}$ ) challenge in the mouse lung, PBS, free TPCA-1 (2 mg/kg), two types of Abs-coated NPs loaded with TPCA-1 (equal to $2 \mathrm{mg} / \mathrm{kg}$ of TPCA-1) were $i . v$. administered. Lung bronchoalveolar lavage fluid (BALF) and lung were collected for analysis at $20 \mathrm{~h}$ postinjection. ${ }^{5}$ Neutrophil numbers and inflammatory factors (IL-6 and TNF- $a$ ) in BALF were significantly decreased after treatment with endothelial targeted nanotherapeutics compared to the controls (Figure 5C-E), indicating that inflammation was efficiently reduced. Protein permeability in the lungs is the hallmark of acute lung inflammation/injury. ${ }^{23}$ As shown in Figure 5F, protein concentrations in BALF were decreased after treatment with endothelial targeted nanotherapeutics in comparison with other formulations. The results indicated that the lung inflammation was resolved and lung vasculature was repaired. Moreover, the histological analysis of lung tissues was studied to evaluate the therapeutic efficacy of various drug formulations (Figure 5G-J). After treatment with endothelial targeted nanotherapeutics (Figure 5J), leukocyte infiltration was significantly decreased compared with the other controls (PBS-, free drug- and non-endothelial targeted nanotherapeutics treatment). In addition, the cytotoxicity of copolymer was evaluated by CCK-8 assay, and the result showed the toxicity of copolymer was negligible (Supporting Information Figure S7). Collectively, endothelial targeted nanotherapeutics can efficiently reduce the lung inflammation, implying that anti-ICAM-1 linked to the surface of NPs increases the drug delivery, thus improving the ALI therapy.

Acute lung inflammation/injury is a serious vascular illness, and in particular the severe form, ARDS can cause the high morbidity and mortality. Care management using ventilation and fluid support is only a means to control the progression of ALI. There are no pharmacological treatments available because specifically delivering of drugs to the inflamed lungs still remains challenging. Great efforts harnessed to develop effective drug delivery systems and efficient delivery strategies to the lungs, but it is lacking to design nanoparticle systems based on the pathogenesis of ALI. The pathogenesis of ALI is associated with the vasculature inflammation and neutrophil-mediated inflammation. During the inflammation, the lung vasculature highly expresses the adhesion molecules, such as ICAM-1. In addition, the inflammation lesions show the low $\mathrm{pH}$. Therefore, we developed a new polymeric micelle comprised of $\mathrm{pH}$-responsive segments and hydrophilic shell, and the micelles were coated with anti-ICAM-1 for lung targeting. Our DPD simulation suggests that the copolymers can self-assemble into micelles, and the core can contain hydrophobic 
drugs, such as TPCA-1 (Figure 2). Interestingly, biotin was exposed on the surface of a micelle, thus allowing the bioconjugation. To prove this idea, we measured the size and morphology of nanoparticles using SDS-PAGE, DLS and TEM, and quantified antibody coating to NPs using BCA assay (Figure 2 and Figure S4). The results suggest that the polymers formed micelles and antibody can be linked to the surface of micelles.

Furthermore, we demonstrated that anti-ICAM-1-coating to NPs is required to promote the binding/uptake of NPs to endothelial cells in vitro (Figure 3A-C). Consistently, in vivo imaging of inflamed mouse lungs (Figure 4) shows that the coating of anti-ICAM-1 is critical to enhance the accumulation of NPs in lung. We also observed that the inflammation condition can support the intercellular trafficking of NPs across a monolayer of endothelium (Figure 3D-E), thus delivering drugs in the airspace of lungs. In the ALI mouse model, we found that nanoparticles coated with targeting ligands (anti-ICAM-1) can dramatically diminish lung inflammation and repair the lung edema, thus preventing the progression of ALI (Figure 5). Together, our data strongly support our design of $\mathrm{pH}$-responsive polymeric nanoparticles used to specifically target inflamed lung vasculature during the pathogenesis of ALI after i.v. administration.

In the current studies, we used biotin-avidin linking to conjugate antibody to the surface of nanoparticle because this approach is very simple and quick to examine our hypothesis in this study. However, immune responses to biotins and avidin in the body may be the issue in translation of our nanoparticle formulation. Many chemical reactions are available for conjugation of anti-ICAM-1 to NPs when our nanoparticles are ready for medical applications.

\section{Conclusion}

In summary, we have demonstrated a new design of $\mathrm{pH}$-responsive drug delivery system made of polymeric micelles loaded with an anti-inflammation agent and coated with lung targeted ligands. This drug delivery system can specifically target inflammatory mouse lungs after intravenous administration and control the drug release triggered by the low $\mathrm{pH}$ existing in inflammatory tissues. The stimuli-responsive linear amphiphilic copolymers are comprised of $\mathrm{pH}$-sensitive and hydrophilic segments, and biotins on the two terminals. We found that this copolymer is able to self-assemble into polymeric micelles loaded with a hydrophobic anti-inflammatory drug and biotin segments are exposed on the surface of PMs available for biofunctionalization. We also showed that our NPs can selectively target inflammatory endothelium and the mouse lungs in the acute lung inflammation model. Consistently, NPs can significantly prevent mouse acute lung inflammation, indicating our drug delivery formulations may be a means to treat ALI. Endothelium is a main component of blood vessel and ICAM-1 is related to vascular inflammation response, therefore our newly-developed bio-responsive nanoparticles may be applied to treating a wide range of vascular inflammation-associated diseases such as cancer and sepsis.

\section{Supplementary Material}

Refer to Web version on PubMed Central for supplementary material. 


\section{Acknowledgements}

This work was supported by National Institute of Health RO1GM116823 to Z.W.

\section{References}

1. Dinarello CA Anti-Inflammatory Agents: Present and Future. Cell 2010, 140 (6), 935-950. [PubMed: 20303881]

2. Nathan C; Ding A Nonresolving Inflammation. Cell 2010, 140 (6), 871-882. [PubMed: 20303877]

3. Matthay MA; Ware LB; Zimmerman GA The Acute Respiratory Distress Syndrome. J. Clin. Invest 2012, 122 (8), 2731-2740. [PubMed: 22850883]

4. Matthay MA; Zemans RL The Acute Respiratory Distress Syndrome: Pathogenesis and Treatment. Annu. Rev. Pathol 2011, 6, 147-163. [PubMed: 20936936]

5. Chu D; Gao J; Wang Z Neutrophil-Mediated Delivery of Therapeutic Nanoparticles across Blood Vessel Barrier for Treatment of Inflammation and Infection. ACS Nano 2015, 9 (12), 11800-11811. [PubMed: 26516654]

6. Wang Z; Li J; Cho J; Malik AB Prevention of Vascular Inflammation by Nanoparticle Targeting of Adherent Neutrophils. Nat. Nanotechnol 2014, 9 (3), 204-210. [PubMed: 24561355]

7. Wang Z; Tiruppathi C; Cho J; Minshall RD; Malik AB Delivery of Nanoparticle: Complexed Drugs Across the Vascular Endothelial Barrier via Caveolae. IUBMB Life 2011, 63 (8), 659-667. [PubMed: 21766412]

8. Wang Z; Tiruppathi C; Minshall RD; Malik AB Size and Dynamics of Caveolae Studied Using Nanoparticles in Living Endothelial Cells. ACS Nano 2009, 3 (12), 4110-4116. [PubMed: 19919048]

9. Secret E; Kelly SJ; Crannell KE; Andrew JS Enzyme-Responsive Hydrogel Microparticles for Pulmonary Drug Delivery. ACS Appl. Mater. Interfaces 2014, 6 (13), 10313-10321. [PubMed: 24926532]

10. Kim S-W; Park J-Y; Lee S; Kim S-H; Khang D Destroying Deep Lung Tumor Tissue through Lung-Selective Accumulation and by Activation of Caveolin Uptake Channels Using a Specific Width of Carbon Nanodrug. ACS Appl. Mater. Interfaces 2018, 10 (5), 4419-4428. [PubMed: 29309112]

11. Su L; Li R; Khan S; Clanton R; Zhang F; Lin Y-N; Song Y; Wang H; Fan J; Hernandez S; Butters AS; Akabani G; MacLoughlin R; Smolen J; Wooley KL Chemical Design of Both a GlutathioneSensitive Dimeric Drug Guest and a Glucose-Derived Nanocarrier Host to Achieve Enhanced Osteosarcoma Lung Metastatic Anticancer Selectivity. J. Am. Chem. Soc 2018, 140 (4), 1438 1446. [PubMed: 29350522]

12. Hussain S; Ji Z; Taylor AJ; DeGraff LM; George M; Tucker CJ; Chang CH; Li R; Bonner JC; Garantziotis S Multiwalled Carbon Nanotube Functionalization with High Molecular Weight Hyaluronan Significantly Reduces Pulmonary Injury. ACS Nano 2016, 10 (8), 7675-7688. [PubMed: 27459049]

13. Gao J; Wang S; Wang Z High Yield, Scalable and Remotely Drug-Loaded Neutrophil-Derived Extracellular Vesicles (EVs) for Anti-Inflammation Therapy. Biomaterials 2017, 135, 62-73. [PubMed: 28494264]

14. Gao J; Chu D; Wang Z Cell Membrane-Formed Nanovesicles for Disease-Targeted Delivery. J. Controlled Release 2016, 224, 208-216.

15. Wang S; Gao J; Wang Z Outer Membrane Vesicles for Vaccination and Targeted Drug Delivery. Wiley Interdiscip. Rev.: Nanomed. Nanobiotechnol 2018, e1523. [PubMed: 29701017]

16. Wang S; Gao J; Li M; Wang L; Wang Z A Facile Approach for Development of a Vaccine Made of Bacterial Double-Layered Membrane Vesicles (DMVs). Biomaterials 2018, 187, 28-38. [PubMed: 30292939]

17. Abdelaziz HM; Gaber M; Abd-Elwakil MM; Mabrouk MT; Elgohary MM; Kamel NM; Kabary DM; Freag MS; Samaha MW; Mortada SM; Elkhodairy KA; Fang J-Y; Elzoghby AO Inhalable Particulate Drug Delivery Systems for Lung Cancer Therapy: Nanoparticles, Microparticles, Nanocomposites and Nanoaggregates. J. Controlled Release 2018, 269, 374-392. 
18. Kim SY; Wong AH; Abou Neel EA; Chrzanowski W; Chan HK The Future Perspectives of Natural Materials for Pulmonary Drug Delivery and Lung Tissue Engineering. Expert Opin. Drug Delivery 2015, 12 (6), 869-887.

19. Cai X; Luo Y; Zhang W; Du D; Lin Y pH-Sensitive ZnO Quantum Dots-Doxorubicin Nanoparticles for Lung Cancer Targeted Drug Delivery. ACS Appl. Mater. Interfaces 2016, 8 (34), 22442-22450. [PubMed: 27463610]

20. Cao H; Dan Z; He X; Zhang Z; Yu H; Yin Q; Li Y Liposomes Coated with Isolated Macrophage Membrane Can Target Lung Metastasis of Breast Cancer. ACS Nano 2016, 10 (8), 7738-7748. [PubMed: 27454827]

21. Kaczmarek JC; Patel AK; Kauffman KJ; Fenton OS; Webber MJ; Heartlein MW; DeRosa F; Anderson DG Polymer-Lipid Nanoparticles for Systemic Delivery of mRNA to the Lungs. Angew. Chem 2016, 128 (44), 14012-14016.

22. Anselmo AC; Kumar S; Gupta V; Pearce AM; Ragusa A; Muzykantov V; Mitragotri S Exploiting Shape, Cellular-Hitchhiking and Antibodies to Target Nanoparticles to Lung Endothelium: Synergy Between Physical, Chemical and Biological Approaches. Biomaterials 2015, 68, 1-8. [PubMed: 26241497]

23. Mehta D; Malik AB Signaling Mechanisms Regulating Endothelial Permeability. Physiol. Rev 2006, 86 (1), 279-367. [PubMed: 16371600]

24. Nathan C, Neutrophils and Immunity: Challenges and Opportunities. Nat. Rev. Immunol 2006, 6, 173-182. [PubMed: 16498448]

25. Grommes J; Soehnlein O Contribution of Neutrophils to Acute Lung Injury. Mol. Med 2011, 17, 293-307. [PubMed: 21046059]

26. Diep BA; Chan L; Tattevin P; Kajikawa O; Martin TR; Basuino L; Mai TT; Marbach H; Braughton KR; Whitney AR; Gardner DJ; Fan X; Tseng CW; Liu GY; Badiou C; Etienne J; Lina G; Matthay MA; DeLeo FR; Chambers HF Polymorphonuclear Leukocytes Mediate Staphylococcus Aureus Panton-Valentine Leukocidin-Induced Lung Inflammation and Injury. Proc. Natl Acad. Sci 2010, 107 (12), 5587-5592. [PubMed: 20231457]

27. Chu D; Dong X; Shi X; Zhang C; Wang Z Neutrophil-Based Drug Delivery Systems. Adv. Mater 2018, 30 (22), e1706245. [PubMed: 29577477]

28. Barton GM A Calculated Response: Control of Inflammation by the Innate Immune System. J. Clin. Invest 2008, 118 (2), 413-420. [PubMed: 18246191]

29. Pober JS; Sessa WC Evolving Functions of Endothelial Cells in Inflammation. Nat. Rev. Immunol 2007, 7 (10), 803-815. [PubMed: 17893694]

30. Podolin PL; Callahan JF; Bolognese BJ; Li YH; Carlson K; Davis TG; Mellor GW; Evans C; Roshak AK Attenuation of Murine Collagen-Induced Arthritis by a Novel, Potent, Selective Small Molecule Inhibitor of IkappaB Kinase 2, TPCA-1 (2-[(aminocarbonyl)amino]-5-(4-

fluorophenyl)-3-thiophenecarboxamide), Occurs via Reduction of Proinflammatory Cytokines and Antigen-Induced T cell Proliferation. J. Pharmacol. Exp. Ther 2005, 312 (1), 373-381. [PubMed: 15316093]

31. Birrell MA; Hardaker E; Wong S; McCluskie K; Catley M; De Alba J; Newton R; Haj-Yahia S; Pun KT; Watts CJ I $x-B$ Kinase-2 Inhibitor Blocks Inflammation in Human Airway Smooth Muscle and a Rat Model of Asthma. Am. J. Respir. Crit. Care Med 2005, 172 (8), 962-971. [PubMed: 16002568]

32. Lo Y-S; Huefner ND; Chan WS; Stevens F; Harris JM; Beebe TP Specific Interactions between Biotin and Avidin Studied by Atomic Force Microscopy Using the Poisson Statistical Analysis Method. Langmuir 1999, 15 (4), 1373-1382.

33. Livnah O; Bayer EA; Wilchek M; Sussman JL Three-Dimensional Structures of Avidin and the Avidin-Biotin Complex. Proc. Natl. Acad. Sci 1993, 90 (11), 5076-5080. [PubMed: 8506353]

34. Kumar V; Singh R; Joshi KB Biotin-Avidin Interaction Triggers Conversion of Triskelion Peptide Nanotori into Nanochains. New J. Chem 2018, 42 (5), 3452-3458.

35. Zhang CY; Gao J; Wang Z Bioresponsive Nanoparticles Targeted to Infectious Microenvironments for Sepsis Management. Adv. Mater 2018, 30 (43), 1803618. 
36. Li J; Ma YJ; Wang Y; Chen BZ; Guo XD; Zhang CY Dual Redox/pH-Responsive Hybrid PolymerLipid Composites: Synthesis, Preparation, Characterization and Application in Drug Delivery with Enhanced Therapeutic Efficacy. Chem. Eng. J 2018, 341, 450-461.

37. Zhang CY; Yang YQ; Huang TX; Zhao B; Guo XD; Wang JF; Zhang LJ Self-Assembled pHResponsive MPEG-b-(PLA-co-PAE) Block Copolymer Micelles for Anticancer Drug Delivery. Biomaterials 2012, 33 (26), 6273-6283. [PubMed: 22695069]

38. Wu D-Q; Lu B; Chang C; Chen C-S; Wang T; Zhang Y-Y; Cheng S-X; Jiang X-J; Zhang X-Z; Zhuo R-X Galactosylated Fluorescent Labeled Micelles as a Liver Targeting Drug Carrier. Biomaterials 2009, 30 (7), 1363-1371. [PubMed: 19100617]

39. Wang Y; Zhu DD; Zhou J; Wang QL; Zhang CY; Liu YJ; Wu ZM; Guo XD Mesoscopic Simulation Studies on the Formation Mechanism of Drug Loaded Polymeric Micelles. Colloids Surf., B 2015, 136, 536-544.

40. Wang Y; Li QY; Liu XB; Zhang CY; Wu ZM; Guo XD Mesoscale Simulations and Experimental Studies of pH-Sensitive Micelles for Controlled Drug Delivery. ACS Appl. Mater. Interfaces 2015, 7 (46), 25592-25600. [PubMed: 26539742]

41. Huang X; Liao W; Zhang G; Kang S; Zhang CY pH-Sensitive Micelles Self-Assembled From Polymer Brush (PAE-g-cholesterol)-b-PEG-b-(PAE-g-cholesterol) for Anticancer Drug Delivery and Controlled Release. Int. J. Nanomed 2017, 12, 2215-2226.

42. Hu YQ; Kim MS; Kim BS; Lee DS RAFT Synthesis of Amphiphilic (A-ran-B)-b-C Diblock Copolymers with Tunable pH-sensitivity. J. Polym. Sci., Part A: Polym. Chem 2008, 46 (11), 3740-3748.

43. Cesur H; Rubinstein I; Pai A; Önyüksel H Self-Associated Indisulam in Phospholipid-Based Nanomicelles: a Potential Nanomedicine for Cancer. Nanomed.: Nanotechnol 2009, 5 (2), 178183. 
A

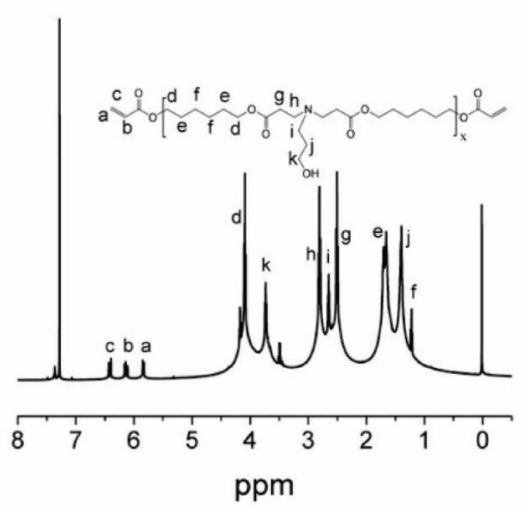

$\mathrm{C}$

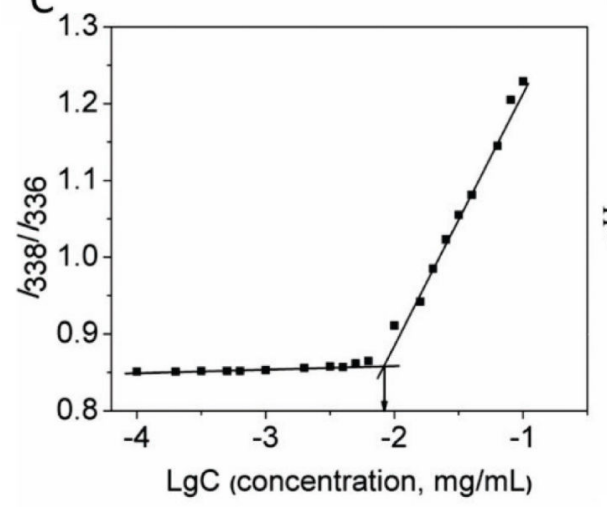

B
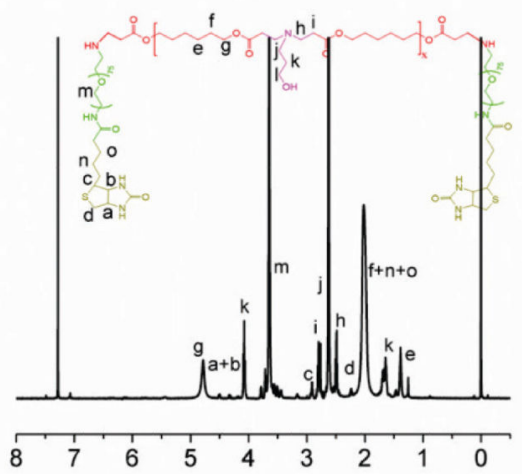

ppm

D

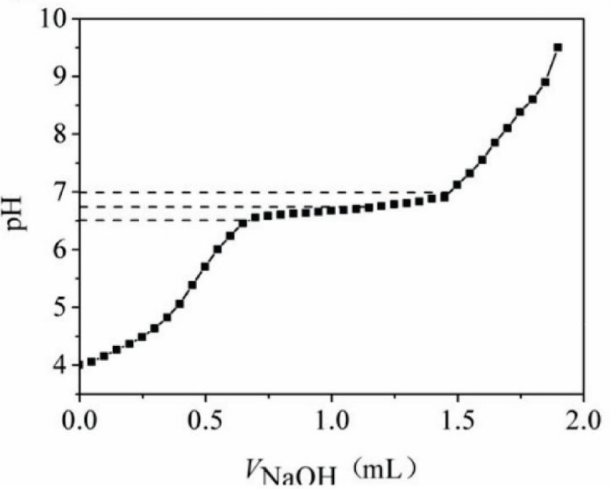

Figure 1. Characterization of polymer and self-assembled polymeric NPs.

${ }^{1} \mathrm{H}-\mathrm{NMR}$ spectra of acrylate-ended PAE (A) and block copolymer $(\mathbf{B})$ in $d-\mathrm{CDCl}_{3}$. C. A plot of intensity ratios $\left(I_{338} / I_{336}\right)$ as function of logarithm of copolymer Biotin-PEG- $b$-PAE- $b$ PEG-Biotin concentrations in aqueous solution. D. The potentiometric titration curve of the copolymer Biotin-PEG- $b$-PAE- $b$-PEG-Biotin solutions. 
A

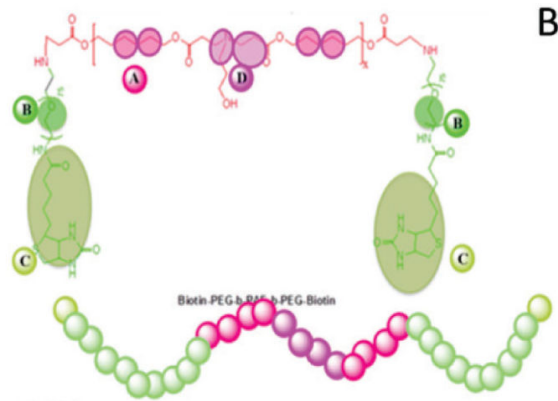

(A) Polymer

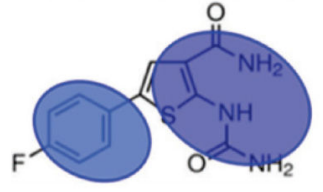

(B) Drug $\mathrm{OO}$

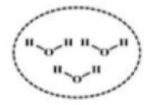

(C) Water $\mathrm{Q}$
B

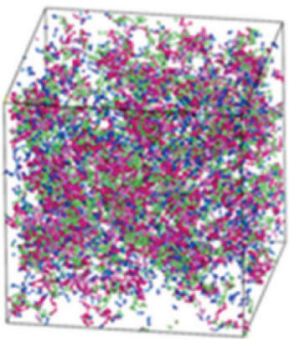

(a) 0 step

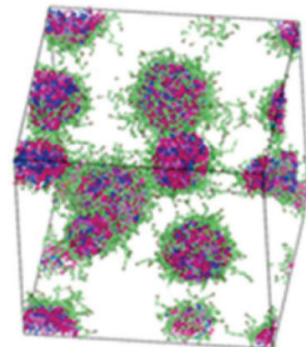

(c) 50000 steps

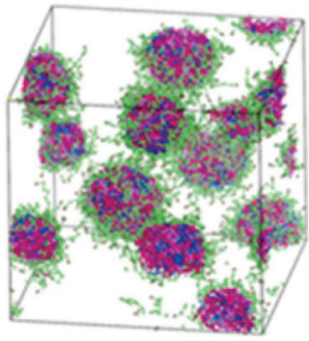

(b) 15000 steps

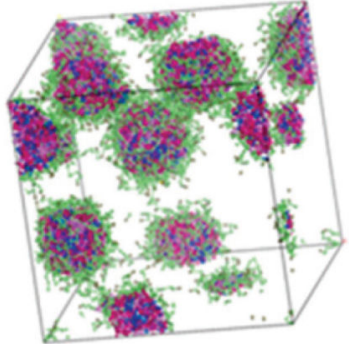

(d) 100000 steps

E

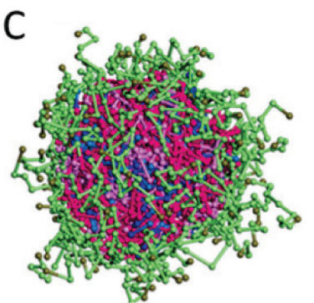

(a) Full-section view

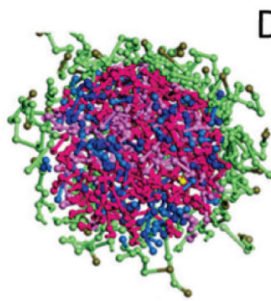

(b) Cross-section view

2

$\mathrm{F}$

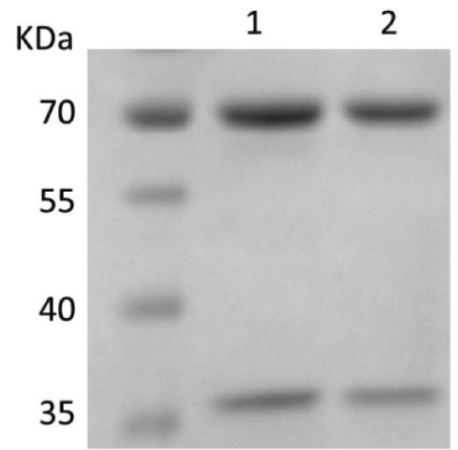

D
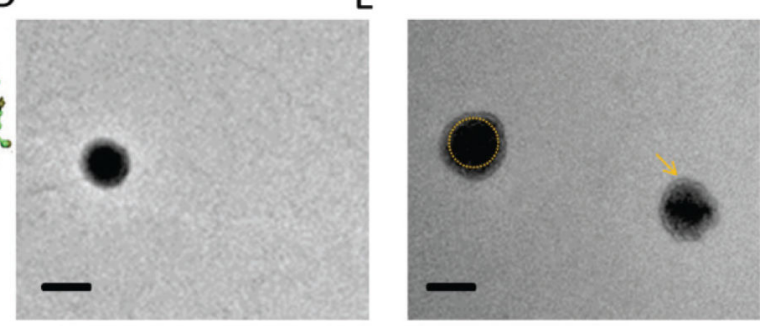

G

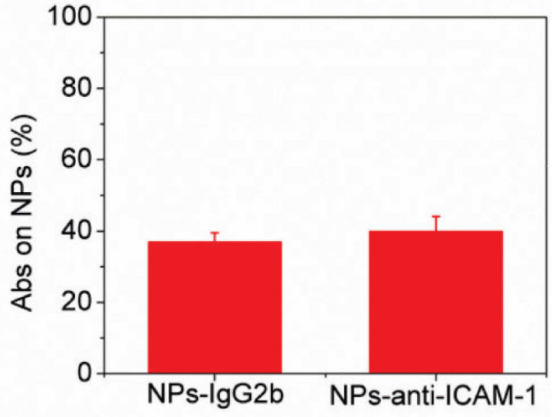

Figure 2. Self-assembly of copolymer and biofunctionalization of PMs.

A. Coarse-grained models of block copolymer, TPCA-1 and water. The copolymer molecule is coarse grained into four types of beads: PEG (light green); biotin (dark green); PAE (pink); TPCA-1 molecule (blue). TPCA-1 is coarse-grained as two beads (blue). Three water molecules are combined as one bead (black). B. Coarse-grained simulation for self-assembly TPCA-1-PMs operated by DPD method. The detail of simulation parameters is shown in Table S1. It clearly shows the formation of PMs after 100,000 steps. C. A full-section view and a cross-section view of TPCA-1-PMs after DPD simulation. TEM images of TPCA-1PMs (D) and TPCA-1-NPs-anti-ICAM-1(E). Scale bars: $100 \mathrm{~nm}$. F. SDS-PAGE analysis of anti-ICAM-1 (lane 1) and anti-ICAM-1-decorated NPs (lane 2). Lane 1, Standard antiICAM-1, $0.5 \mathrm{mg} / \mathrm{mL}$; Lane 2, NPs-anti-ICAM- $1,2.5 \mathrm{mg} / \mathrm{mL}$. G. Antibody coating efficiency on NPs quantified by BCA assays. 

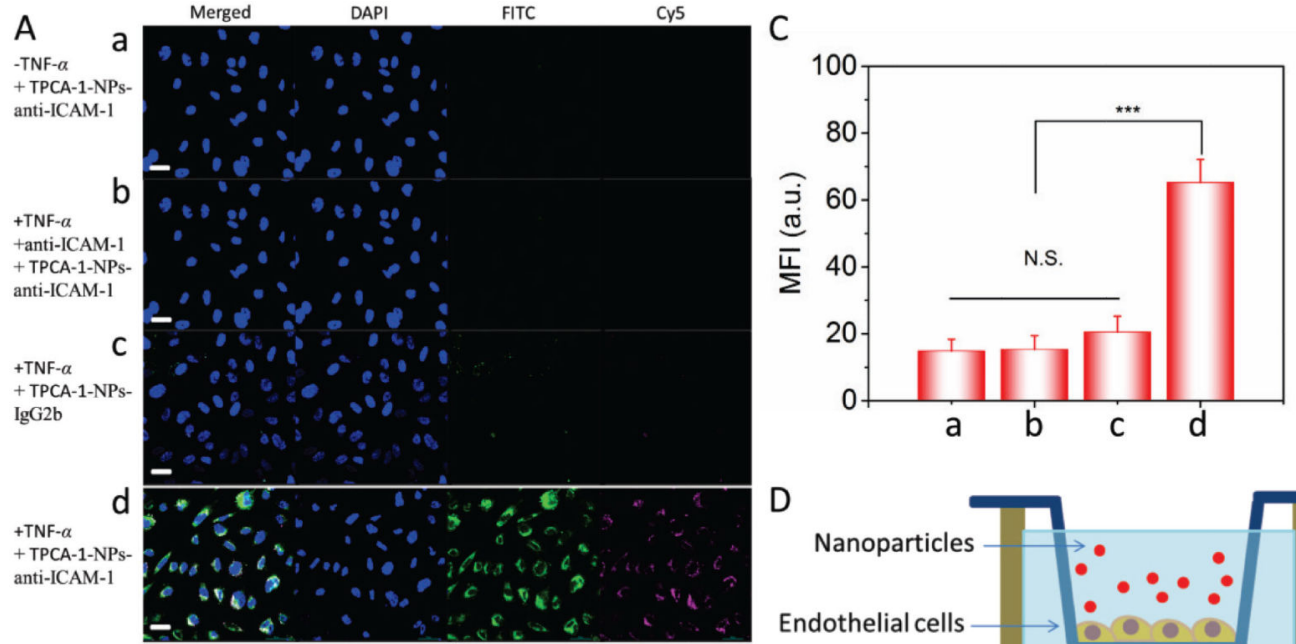

B
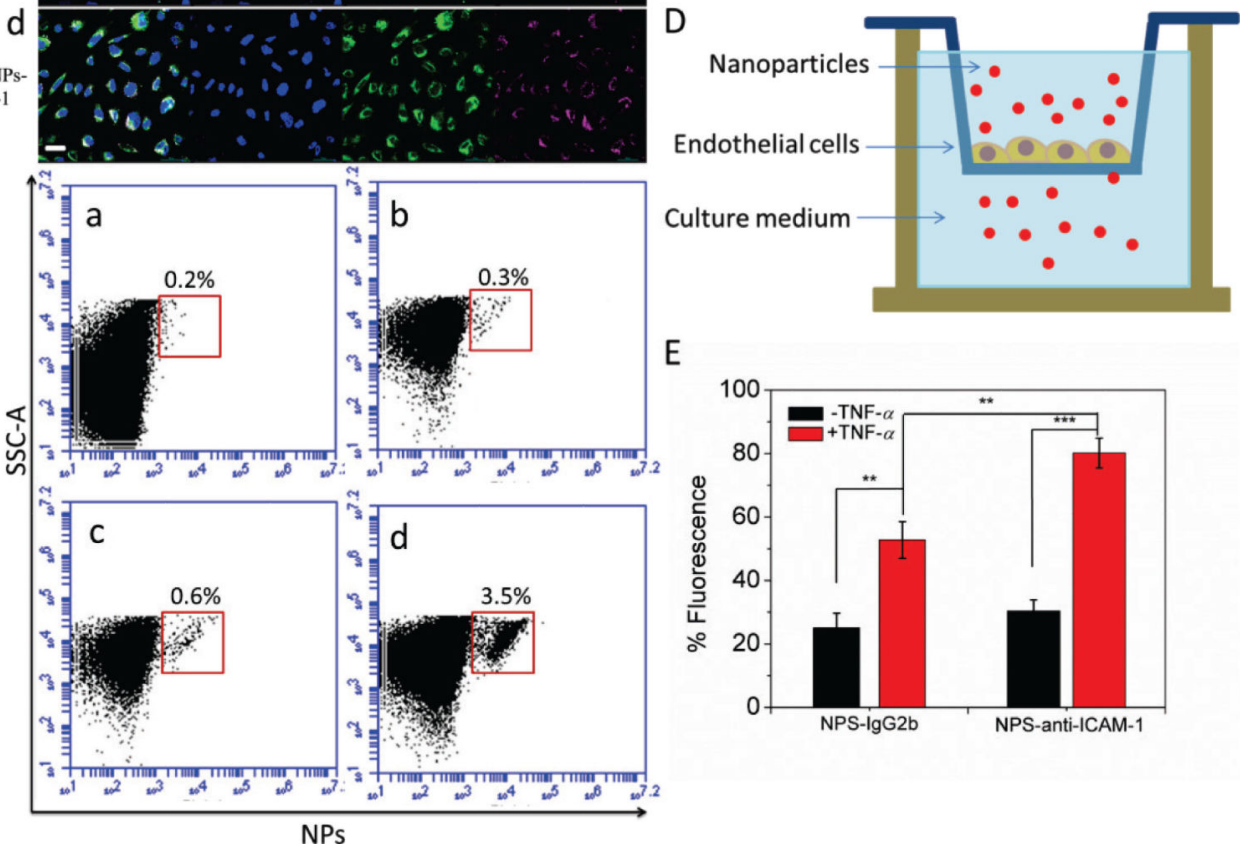

$\mathrm{E}$

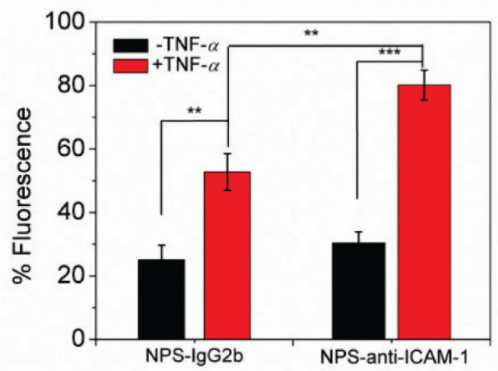

Figure 3. Endothelial targeted nanotherapeutics selectively bind to activated endothelia and deliver drugs in vitro.

A. Confocal laser scanning microscopy (CLSM) images show interactions between HUVECs and NPs. a) HUVECs were treated with endothelial targeted nanotherapeutics, b) TNF- $a$-activated HUVECs were treated with anti-ICAM-1, followed by incubation with endothelial targeted nanotherapeutics, c) TNF-a-activated HUVECs were treated with nonendothelial targeted nanotherapeutics, d) TNF-a-activated HUVECs were treated with endothelial targeted nanotherapeutics. Scale bars: $201 / 4 \mathrm{~m}$. B. Quantitative analysis of HUVECs interacting with NPs using flow cytometry. C. MFI of HUVECs with different treatment analyzed by flow cytometry after incubation with NPs. D. Diagram shows an assay to test transport of nanoparticles across a monolayer of endothelia using Transwell. E. FITC-labeled NPs emission in the lower chambers of a Transwell system. The \% fluorescence means the ratio of fluorescence of NPs in the lower chamber over the fluorescence of total NPs. Plots show means \pm s.d. $(\mathrm{n}=3) . P$ values: $* * p<0.01, * * * p<$ 0.001 . 
A
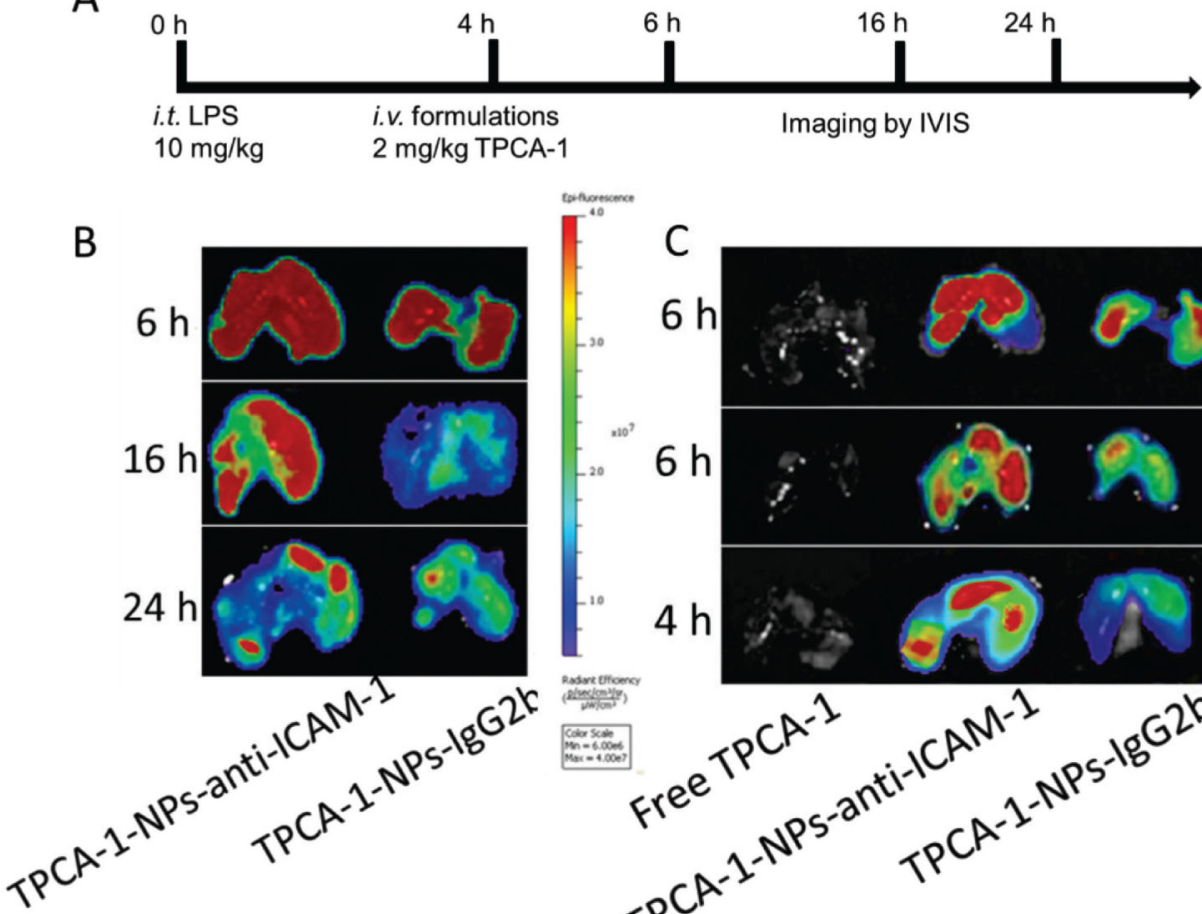

D

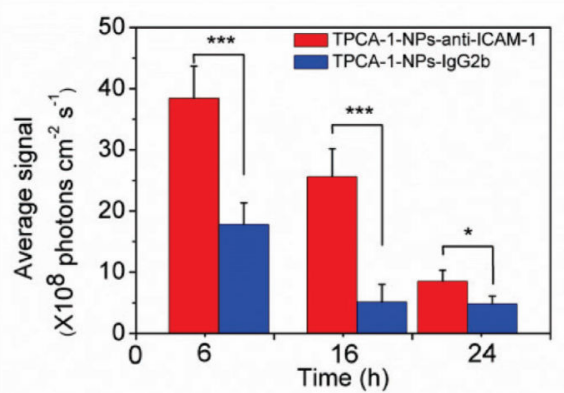

C

$6 \mathrm{~h}$
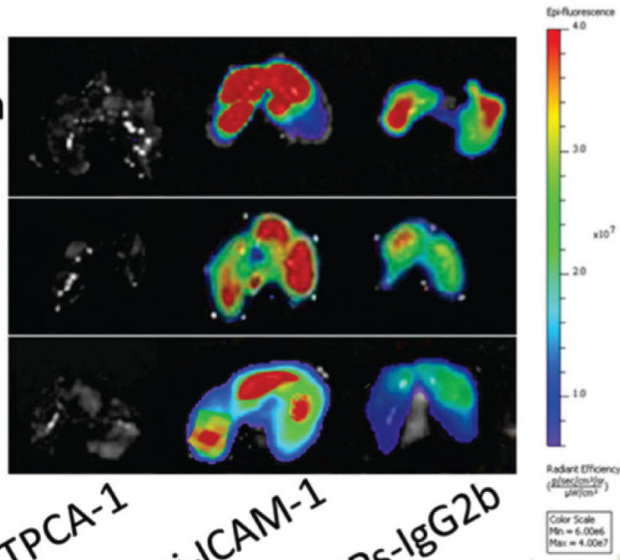

Figure 4. Endothelial targeted nanotherapeutics selectively deliver drugs to the site of inflammation.

A. Experimental protocol for evaluating the targeting of NPs to inflammatory mouse lungs. Acute lung inflammation mouse model was generated by intratracheal (i.t.) administration of LPS (15 mg/kg). $4 \mathrm{~h}$ after LPS challenge, fluorescent Abs-coated TPCA-1-NPs were intravenously administered. The lungs were collected for imaging. The fluorescence of FITC-labeled NPs (B) and Cy5-labeled TPCA-1 (C) was measured using IVIS, respectively. Quantitative analysis of NPs (D) and TPCA-1 (E) in lungs confirmed by IVIS. The data are shown as mean \pm s.d. $(\mathrm{n}=3)$. $P$ values: $* p<0.05, * * * p<0.001$. 
A

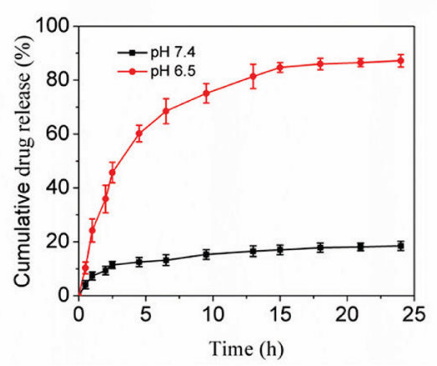

$\mathrm{C}$

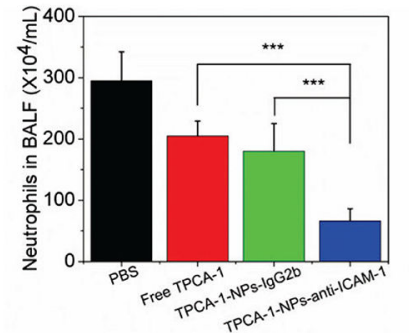

$\mathrm{E}$
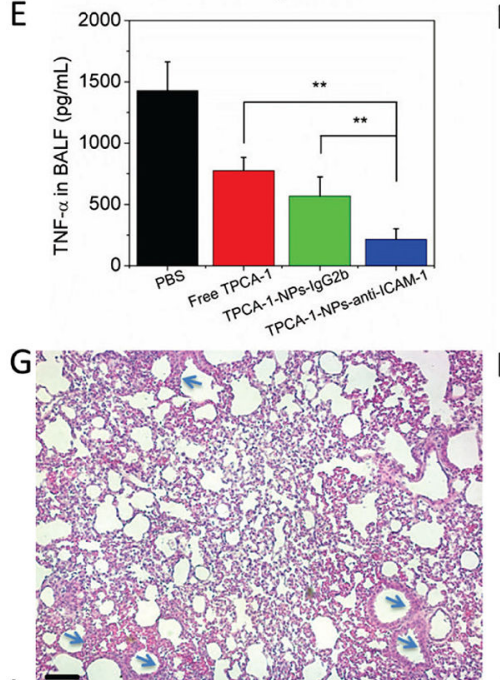

I

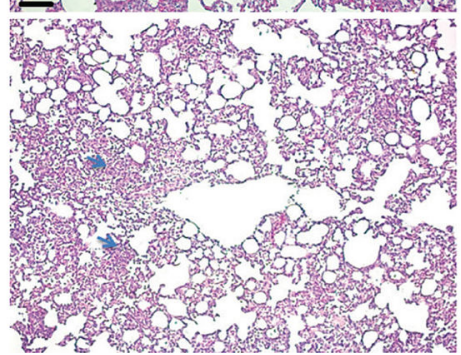

B

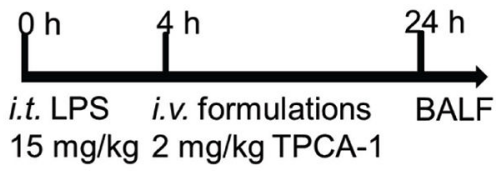

D

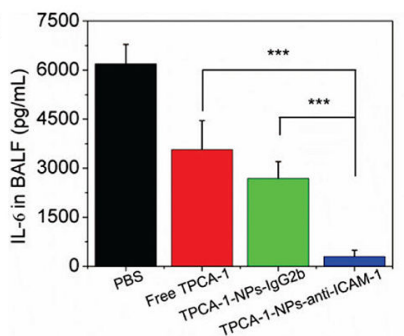

$\mathrm{F}$
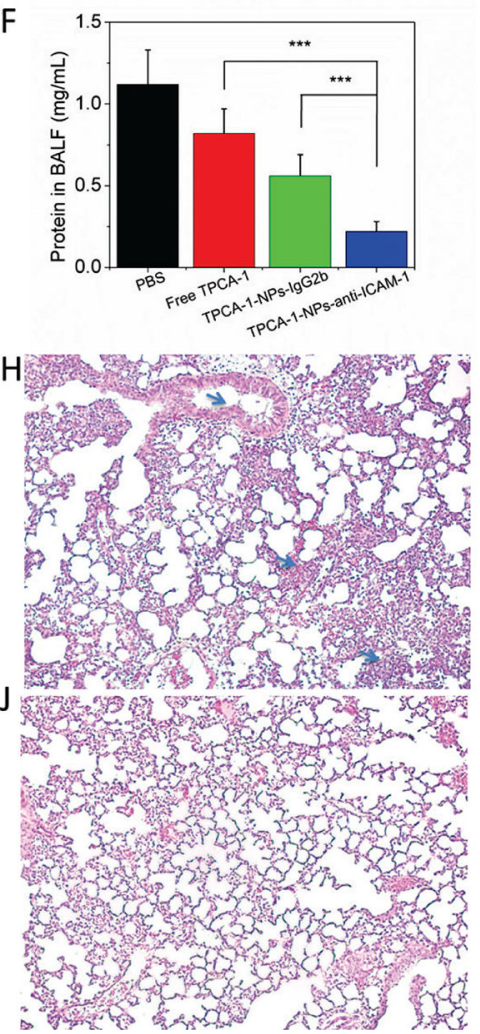

Figure 5. Endothelial targeted nanotherapeutics can improve therapeutic efficacy of ALI in a mouse model.

A. In vitro release of TPCA-1 from endothelial targeted nanotherapeutics in PBS at different pH. B. Experimental protocol for evaluating therapeutic effect of free drug, non-endothelial targeted nanotherapeutics and endothelial targeted nanotherapeutics. PBS treatment was used as control. Acute lung inflammation mouse model was generated by intratracheal (i.t.) administration of LPS $(15 \mathrm{mg} / \mathrm{kg}) .4 \mathrm{~h}$ after LPS-challenge, the mouse was treated with different drug formulations. $20 \mathrm{~h}$ post-administration of therapeutics, the mouse lungs and 
BALF were collected for therapeutic analysis. Neutrophil numbers (C), IL-6 (D), TNF- $a$ (E) and protein contents $(\mathbf{F})$ in BALF were measured using a hemocytometer and ELISA. H\&E-stained lung tissue sections were imaged. PBS $(\mathbf{G})$, free drug $(\mathbf{H})$, non-endothelial targeted nanotherapeutics (I) and endothelial targeted nanotherapeutics $(\mathbf{J})$. The blue arrows indicate neutrophil infiltration. Scale bar: $100 \mu \mathrm{m}$. The data are shown as mean \pm s.d. ( $\mathrm{n}=$ 5). $p$ values: $* * p<0.01, * * * p<0.001$. 


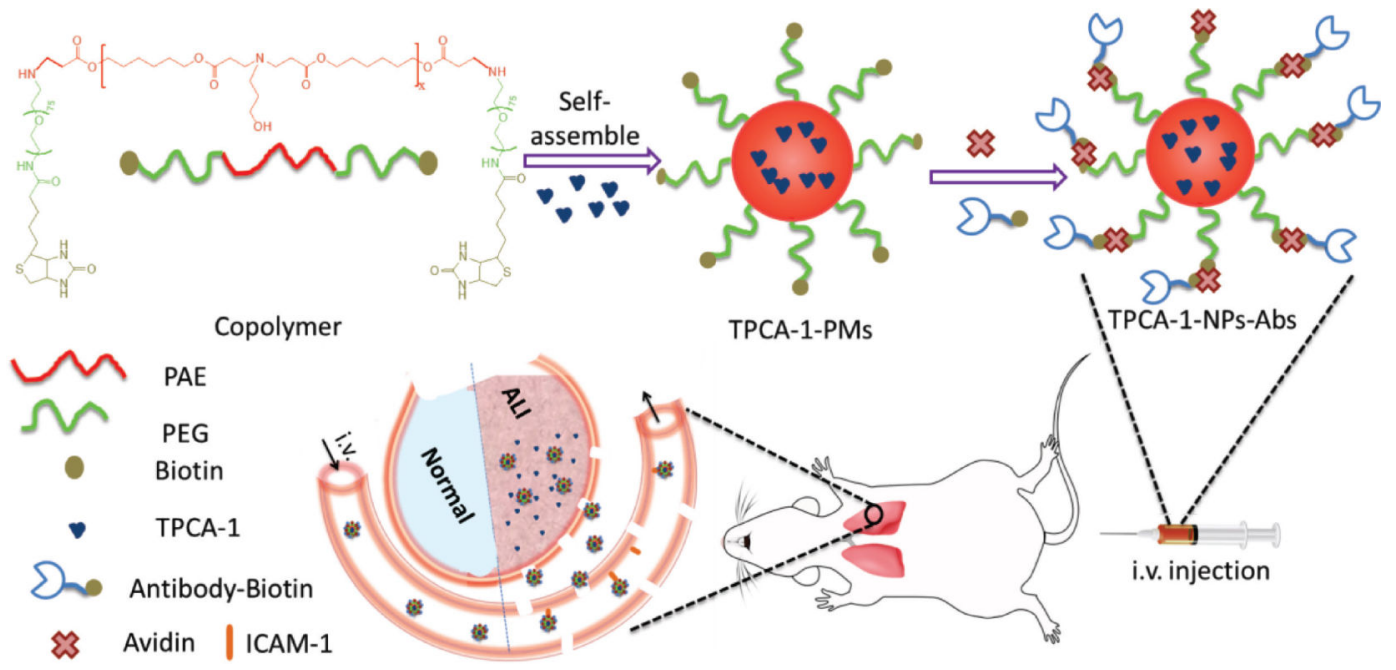

Scheme 1. Illustration of development of an antibody-decorated nanoparticle targeted to mouse lungs for therapy of acute lung inflammation.

PAE copolymers self-assemble to form a micellar nanoparticle loaded with TPCA-1,

followed by decorating antibodies via the interaction between biotin and avidin. In the acute lung inflammation mouse model, endothelial targeted nanotherapeutics targets to inflamed lung endothelial cells, and subsequently release the drug triggered by low $\mathrm{pH}$ existing in inflammatory tissues after i. v. administration. The enlarged figure of lung tissue shows the alveolar structure of the mouse lung. In the normal physiology, the alveolae form the integrity of blood vessels, and nanoparticles cannot transport into the alveolae. However, in the acute lung inflammation, the vessel permeability is dramatically increased and endothelium highly expresses ICAM-1, thus NPs coated with anti-ICAM-1 are able to target inflamed vasculature and may transport into alveolar air space. Low $\mathrm{pH}$ in inflamed lungs promotes the drug release from NPs. 